Repository of the Max Delbrück Center for Molecular Medicine (MDC) Berlin (Germany)

\title{
Identification of the mitochondrial MSRB2 as a binding partner of LG72
}

Otte, D.M., Rasko, T., Wang, M., Dreiseidler, M., Drews, E., Schrage, H., Wojtalla, A., Hoehfeld, J., Wanker, E., Zimmer, A. 


\section{Identification of the mitochondrial MSRB2 as a binding partner of LG72}

David-Marian Otte ${ }^{1 *}$, Tamás Raskó*2, Mengzhe Wang ${ }^{1}$, Michael Dreiseidler ${ }^{3}$, Eva Drews ${ }^{1}$, Hanna Schrage ${ }^{1}$, Alexandra Wojtalla ${ }^{1}$, Jörg Höhfeld ${ }^{3}$, Erich Wanker ${ }^{2}$, and Andreas Zimmer ${ }^{1}$

1 Institute of Molecular Psychiatry, University of Bonn, Germany; ${ }^{2}$ Proteomics and Molecular Mechanisms of Neurodegenerative Diseases, Max Delbrück Center for Molecular Medicine Berlin, Germany; ${ }^{3}$ Institute for Cell Biology; University of Bonn, Germany

${ }^{*}$ Authors contributed equally to this work

\section{Corresponding authors:}

Andreas Zimmer

Institute of Molecular Psychiatry

University of Bonn

Sigmund Freud-Str. 25

53125 Bonn, Germany

Tel.: +492286885300

Fax: +492286885302

Email: a.zimmer@uni-bonn.de

Erich Wanker

MDC Max-Delbrück-Centrum, Neuroproteomics

Robert-Rössle-Straße 10

13125 Berlin

Telefon: +49-30/94 06-2157

Telefax: +49-30/94 06-2552

Email: ewanker@mdc-berlin.de 


\begin{abstract}
Genetic studies have linked the evolutionary novel, anthropoid primate-specific gene locus G72/G30 in the etiology of schizophrenia and other psychiatric disorders. However, the function of the protein encoded by this locus, LG72, is currently controversially discussed. Some studies have suggested that LG72 binds to and regulates the activity of the peroxisomal enzyme D-amino-acid-oxidase, while others proposed an alternative role of this protein due to its mitochondrial location in vitro. Studies with transgenic mice expressing LG72 further suggested that high levels of LG72 lead to an impairment of mitochondrial functions with a concomitant increase in reactive oxygen species (ROS) production. In the present study we now performed extensive interaction analyses and identified the mitochondrial methionine-Rsulfoxide reductase B2 (MSRB2) as a specific interaction partner of LG72. MSRB2 belongs to the MSR protein family and functions in mitochondrial oxidative stress defense. Based on our results we propose that LG72 is involved in the regulation of mitochondrial oxidative stress.
\end{abstract}

\title{
Keywords:
}

Schizophrenia, protein interaction analysis, MSR protein family, oxidative stress 


\section{Introduction}

The 13q33.2 chromosomal region has been associated with schizophrenia and bipolar disorder in several independent linkage and association studies (Chumakov et al. 2002; Korostishevsky et al. 2006; Schumacher et al. 2004; Zhang et al. 2009). The prime candidate gene in this chromosomal locus is G72, which is only found in anthropoid primates. It is thought that the disease symptoms, such as impaired cognitive functions (Goldberg et al. 2006), increased progression of prodromal syndromes (Mossner et al. 2010) and grey matter reductions (Zuliani et al. 2009), involve an activation of G72, because expression of G72 mRNA is up-regulated in schizophrenic patients and LG72 protein levels were increased (Korostishevsky et al. 2006).

The longest open reading frame of the locus encodes for a protein, LG72, which represents an exceptional case of a primate-specific protein with a rapidly changing structure (Kvajo et al. 2008). The function of LG72 is still controversially discussed. Some studies have suggested that LG72 binds to the peroxisomal enzyme D-aminoacid-oxidase (DAO). Since both enhancement (Chumakov et al. 2002) and repression (Sacchi et al. 2008) of DAO activity have been reported, the effects of LG72 on peroxisomal DAO functioning are ambiguous. An alternative role for LG72 protein was suggested, as it contains an $\mathrm{N}$-terminal mitochondrial translocation sequence and is transported into mitochondria. Here, LG72 promotes mitochondrial fragmentation in mammalian cell lines and primary neurons (Kvajo et al. 2008) contributing to mitochondrial and cell dysfunction.

To investigate the function of LG72 in vivo, we have generated transgenic G72 (G72Tg) mice that express LG72 and other human splice variants (Otte et al. 2011). Most strikingly, these transgenic animals showed a variety of behavioral phenotypes relevant to schizophrenia and displayed alterations in mitochondrial functions. Thus, we observed a lower activity of the respiratory chain complex I and of the enzyme aconitase, which catalyzes the isomerization of citrate to isocitrate in the citric acid cycle. We also found higher levels of oxidized lipids, reduced levels of glutathione and increased expression of GSH-S-transferase (GST) in G72Tg mice. Together these findings suggest that high levels of LG72 lead to an impairment of mitochondrial functions with a concomitant increase in reactive oxygen species (ROS) production. Importantly, mitochondrial dysfunctions have been associated with 
psychiatric diseases, including schizophrenia (Altar et al. 2005; Clay et al. 2011; Marchbanks et al. 2003; Prabakaran et al. 2004).

These findings prompted us to look for potential mitochondrial binding partners of LG72. Therefore, we performed extensive interaction analyses and identified the mitochondrial methionine sulfoxide $S$ reductase B2 (MSRB2) as a specific interaction partner of LG72. MSRB2 is part of the mitochondrial MSR system, which reduces methionine sulfoxide back into methionine and is thus one of the systems involved in the elimination of cellular ROS. Based on our results we propose that LG72 is involved in the regulation of mitochondrial oxidative stress.

\section{Materials and Methods}

\section{Materials}

HEK-293 and COS cells were purchased from ATCC (Manassas, VA). Dulbecco's modified Eagle's medium (DMEM), the streptomycin/penicillin mix and sodium pyruvate were purchased from GIBCO. Heat-inactivated fetal bovine serum (FBS) and Hank's Buffered Salt Solution (HBSS) was from PAA. The Myc-DDK-tagged ORF clone of Homo sapiens LG72 transcript variant 1 (RC212162, GenBank accession number: NM_172370.2, NP_758958) was from OriGene Technologies (Rockville, MD). X-tremeGENE 9 DNA Transfection Reagent was purchased from Roche Applied Science. The myc antibody was obtained from Abcam. The LG72 antibody (G72(N15):sc-46118) was purchased from Santa Cruz Biotechnology. Rabbit anti-Renilla antibody was purchased from Biocompare. The MSRB2 antibody (T1895) was obtained from Epitomics.

\section{Yeast two-hybrid screen}

A yeast two-hybrid $(\mathrm{Y} 2 \mathrm{H})$ screen was performed to screen for potential LG72 binding partners. We used the GATEWAY technology (Invitrogen) for subcloning of human LG72 full-length cDNAs gene into different type of expression plasmids. For the $\mathrm{Y} 2 \mathrm{H}$ interaction screening, LG72 cDNA (BC121091.1; CCSB_56740) was subcloned into the pBTM-D9 Y2H expression bait plasmid and then transformed into the L40ccua MATa yeast strain. In the array mating process, the non-self-activating $\mathrm{Y} 2 \mathrm{H}$ clone encoding the LG72 bait protein was mated against a prey-matrix of MATa yeast clones, which expressed 16,825 human proteins from the pACT4-DM vector. The 
automated $\mathrm{Y} 2 \mathrm{H}$ screenings were repeated 4 times in order to obtain a high-coverage protein interaction network. Positive interactions activated the reporter genes (His, Ura) and were thus identified by growth on selective agar plates and LacZ reporter activation in ß-galactosidase assays (Stelzl et al. 2005). To select high quality interactions, we developed a confidence scoring system using experimental reproducibility (at least 3 times repeated growth of yeast colonies on selective media and repeated activation of the LacZ reporter).

\section{LUMIER-Assay}

To further assess the quality of the $\mathrm{Y} 2 \mathrm{H}$ interactions, we established a modified version of the LUMIER method, which allows the detection of protein-protein interactions in mammalian cells (Fig.1a (Palidwor et al. 2009; Petrakis et al. 2012)). Fig. 1a outlines the experimental setup. The cDNA encoding the MSRB2 protein as prey was subcloned into the pFireflyV5 LUMIER vector using the GATEWAY system. Additionally, the LG72 protein was subcloned into the plasmid pPARenilla for the use as bait. For the test procedure, ProteinA-Renilla and FireflyV5-luciferase-tagged fusion proteins were co-expressed in HEK-293 cells using transfection (TF) and protein complexes were isolated from cell extracts using IgG-coated microtiter plates. For the control experiment, the pPARenilla plasmid was transfected together with FireflyV5-luciferase-tagged MSRB2 and processed as the test sample. The interaction between bait (PA-RL fusion) and prey protein ( $F L$ fusion) were monitored by quantification of firefly luciferase activities. Quantification of Renilla luciferase activity was used to confirm that PA-RL-tagged bait proteins are successfully immunoprecipitated from cell extracts. To detect Renilla- and firefly luciferase-based luminescence in the samples, the Dual-Glo Luciferase Kit (Promega) was used and bioluminescence was quantified in a luminescence plate reader (TECAN Infinite M1000). Based on empirical studies with a set of well-characterized positive and negative interaction pairs we defined that binding ratios of R-output prey (op) $\geq 1.2$ are indicative of specific interactions. R-op ratios are calculated as the quotient of the firefly luciferase signal (FLS) of the test sample and the FLS of the control sample: R$o p=F L S($ Test $) / F L S($ Control) $\geq 1.2$. To further confirm the binding results of the first experiment, bait and prey were switched and the LUMIER-assay was repeated. 
HEK-293, N2a and COS cells were cultured in DMEM supplemented with $10 \%$ FCS, a streptomycin/penicillin mix and pyruvate at $37^{\circ} \mathrm{C}$ with $5 \% \mathrm{CO} 2$.

\section{Transient lipotransfection}

Following the procedure described for X-tremeGENE 9 DNA Transfection Reagent (Roche Applied Science), $10^{5}$ HEK-293, COS or N2a cells were seeded in 24-well plates, transfected with $1 \mu \mathrm{g}$ DNA and assayed after $24 \mathrm{~h}$. DMEM with 5\% FBS and $1 \%$ sodium pyruvate served as medium for transfection.

\section{Western blotting}

Cells were collected from 6- or 24- wells plates for Western blot analysis. Subsequently, they were washed with phosphate-buffered saline (PBS) and lysed on ice for $40 \mathrm{~min}$ in lysis buffer containing HEPES (4-(2-hydroxyethyl)-1piperazineethanesulfonic acid) buffer $\mathrm{pH}$ 7.5, $10 \mathrm{mM}$ EDTA, 0.1\% NP-40 and complete protease inhibitor cocktail (Roche) as recommended by the manufacturer. Total lysates were resolved by $4-12 \%$ gradient SDS-PAGE (Invitrogen) and transferred to PVDF membranes by semi-dry blotting. The membranes were blocked with TBS-T (TBS supplemented with $0.1 \%$ Tween-20) containing $1 \%$ nonfat dry milk and were then incubated overnight at $4{ }^{\circ} \mathrm{C}$ with primary antibodies in appropriate dilutions in TBS-T containing $1 \%$ nonfat dry milk. Membranes were washed with TBS-T, incubated for $1 \mathrm{~h}$ at room temperature with alkaline phosphatase-conjugated (Sigma-Aldrich) or horseradish peroxidase-conjugated (Promega) secondary antibodies in TBS-T containing 1\% nonfat dry milk (1:10,000). After that, blots were washed with TBS-T. Bands were detected by alkaline phosphatase activity-based luminescence by using the AttoPhos ${ }^{\circledR}$ substrate (Promega) or Immun-Star HRP Chemiluminescence Kit (Bio Rad).

\section{Generation of expression vectors}

The MSRB2-mCherry and MSRB2-GFP constructs were generated using the GATEWAY system. Briefly, the entry plasmid encoding CDNA of human MSRB2 (BC117471; CCSB54196) protein was mixed with the pDEST-mCherry empty vector (Invitrogen) at a ratio of $1: 2$ in the presence of $L R$ clonase $®$ mix as recommended by the manufacturer (Invitrogen). After incubation for $2 \mathrm{~h}$ at $25^{\circ} \mathrm{C}$, the resulting DNA plasmids were transformed into competent Mach1 E. coli cells (Invitrogen). E. coli 
clones were selected on LB agar plates supplemented with ampicillin and were analyzed by BsrGI restriction digestion (NEB). The pCMV-LG72-GFP construct was cloned as described elsewhere (Otte et al. 2011). Briefly, the LG72 open reading frame (identical to the published GenBank sequence AY138546, which results in the longest predicted protein) was amplified from a cerebellum cDNA preparation of G72Tg1 mice by PCR. In order to obtain C-terminally GFP-tagged LG72 in a mammalian expression vector, LG72 cDNA was cloned in frame via Xhol and HindIII restriction sites into the pEGFPN1 vector (Clontech, Saint-Germain-en-Laye France). The construct was verified by sequencing.

\section{Co-Immunoprecipitation}

Cell lysates of HEK-293 cells transiently transfected with LG72-FLAG were prepared by scraping cells from a 10-cm dish with $1.2 \mathrm{ml}$ of ice-cold RIPA buffer (50 mM Tris$\mathrm{HCl}, \mathrm{pH} 7.4,150 \mathrm{mM} \mathrm{NaCl}, 5 \mathrm{mM}$ EDTA, $5 \mathrm{mM}$ EGTA, 0.1\% Triton, Roche Mini EDTA-free proteinase inhibitor cocktail). The lysates were collected into a $1.5 \mathrm{ml}$ Eppendorf tube on ice. Cells were homogenized by passing through a $27 \mathrm{G}$ needle. After 20 min incubation on ice, the soluble and insoluble fractions were separated by centrifugation at $20,000 \mathrm{~g}$ for $20 \mathrm{~min}$ at $4^{\circ} \mathrm{C}$. Protein concentration of the supernatant was determined by the Bradford assay. The supernatant was collected into a fresh tube. Twenty $\mu$ l of FLAG M2 Matrix (Sigma) were added and incubated at $4^{\circ} \mathrm{C}$ for $3 \mathrm{~h}$ under shaking conditions to immunoprecipitate the FLAG-tagged protein and its interaction partners. The immunoprecipitate was sedimented at $2000 \mathrm{~g}$ at $4^{\circ} \mathrm{C}$ for 2 min and the supernatant was discarded. The matrix was washed seven times with 1 $\mathrm{ml}$ Tris-buffered saline. The FLAG-tagged protein and its interaction partners were recovered from the matrix by adding non-reducing SDS buffer and incubating at $95^{\circ} \mathrm{C}$ for $5 \mathrm{~min}$. The proteins were separated by SDS-PAGE and MSRB2 was detected by Western blot analysis using a human MSRB2-specific antibody (Epitomics).

\section{Immunofluorescence}

HEK-293, COS and N2a cells were seeded onto round $13 \mathrm{~mm}$ coverslips. Twentyfour hours after transfection, cells were washed once with HBSS, then fixed for 20 min with $0.5 \mathrm{ml} \mathrm{3 \%}$ paraformaldehyde (PFA) and washed three times with HBSS. For the detection of mitochondrial localization of LG72 and MSRB2 the mitochondrial MitoTracker ${ }^{\circledR}$ (H2MRos) was added to the medium in a final concentration of $0.2 \mathrm{nM}$ 
for $45 \mathrm{~min}$. After this pulse, the cells were cultivated in growth medium without dye for $10 \mathrm{~min}$ before fixation. For immunofluorescence, the cells were incubated for 10 min in quenching solution (50 mM NH4Cl-PBS), then permeabilized in $0.1 \%$ Triton $\mathrm{X}$ 100 PBS for 5 min. After washing three times with HBSS, the cells were blocked in $10 \%$ horse-serum-PBS for $1 \mathrm{~h}$, followed by $1 \mathrm{~h}$ incubation in primary antibody (goat $\alpha$ c-myc, Abcam 1:1000) diluted in the blocking buffer. Next, cells were washed three times in $0.1 \%$ BSA-PBS for 5 min before a $1 \mathrm{~h}$ incubation in the secondary antibody diluted in PBS. After three times washing with HBSS, the coverslips were shortly rinsed in MilliQ H2O, dried and placed onto a microscope slide in $20 \mu \mathrm{l}$ fluoromountG medium (Southern Biotech). Visualization of transfected HEK-293 and COS cells was performed using a Zeiss Axiolmager.M2 microscope and the software AxioVision Rel. 4.8. The objective PL APO 63x 1.4 Oil M27 was used. N2a cells were visualized using a confocal laser scanning microscope (LSM) TCS SP8 (Leica) with HCX PL APO 40x/1.10 W water objective (Leica). Excitation wavelength for the fluorophores (DAPI, eGFP, mCherry, MitoTracker) was 405, 488 and $594 \mathrm{~nm}$, respectively. For detection of the emission signal at specified ranges, the normal photomultiplier (DAPI) or the hybrid GaAsP detector SP (eGFP, mCherry and MitoTracker) were used. LCS (Leica) software was used for image acquisition.

\section{Results}

Interaction of LG72 and MSRB2

We identified the mitochondrial protein MSRB2 as a binding partner of LG72 using a yeast two-hybrid screen. The full dataset of LG72 interaction partners will be published in an independent study. We detected this interaction in four independent screens and additionally confirmed this finding by cell growth on selective media (SD4) and LacZ reporter activation. In contrast, we did not find the DAO protein as interaction partner of LG72 using this high-coverage $\mathrm{Y} 2 \mathrm{H}$ approach. The specificity of this interaction was further confirmed with a modified version of the LUMIER method (Palidwor et al. 2009), allowing the detection of protein-protein interactions in mammalian cells (here: HEK-293 cells) (Fig 1a). Fig. 1b shows interactions determined by quantification of firefly luciferase luminescence from coimmunoprecipitated protein complexes. We found that LG72 specifically interacts 
with MSRB2, indicated by a prey-binding ratio (R-op) above 1.2 (Petrakis et al. 2012). In an additional experiment we switched bait and prey (PA-RL-MSRB2 + FL-LG72) before the LUMIER-assay was repeated. Again, the prey-binding ratio exceeded the interaction threshold of 1.2 (dotted line). Values exceeding this threshold indicate specific protein interaction, as confirmed by former empirical studies (Petrakis et al. 2012).

The next experiment aimed to determine if the endogenously expressed MSRB2 could be precipitated with an antibody directed against the LG72 protein. Therefore a FLAG-tagged LG72 was expressed in HEK-293 cells and an immunoprecipitation with an anti-FLAG antibody, followed by Western blot analysis with an MSRB2specific antibody was performed. As shown in Fig. 1c, a MSRB2- specific $20 \mathrm{kDa}$ band was only detected in the sample transfected with LG72-FLAG, but not in the control sample transfected with an empty vector. These findings strongly support the notion that endogenous MSRB2 is a binding partner for LG72 in HEK-293 cells.

\section{LG72 co-localizes with MSRB2 in COS, HEK-293 and N2a cells}

To investigate the subcellular localization of LG72 and MSRB2, COS and HEK-293 cells were transfected with CMV-MSRB2-mCherry, together with CMV-G72-GFP (Fig. 2a and c) or CMV-G72-myc-flag constructs (Fig. 2b-d). The LG72-GFP and MSRB2mCherry transfected cells were visualized directly, while the myc-flag-tagged LG72 was detected using an anti-myc antibody followed by FITC-linked secondary antibody. Both signals from LG72-GFP or LG72-myc, respectively, co-localized with MSRB2mCherry signals in punctuated to tubular structures within in HEK-293 and COS cells (Fig. 2a-d). To verify the localization of LG72 and MSRB2 in neurons cells, N2a cells were transfected as described for COS and HEK-293 cells. Additionally, to assess the localization of MSRB2 in neuronal cells, an MSRB2-GFP construct was used (Fig. 3a). MSRB2-GFP and LG72-GFP predominantly localize to mitochondria in N2a cells, as shown by co-localization with a mitochondria-specific marker (MitoTracker $\circledast$, Fig. 3.a/b). Furthermore, N2a cells transiently co-transfected with MSRB2-mCherry and LG72-GFP constructs display a high proportion of co-localization (Fig. 3c), indicating the presence of both proteins in the same compartment also in neuronal-like cells. 


\section{Discussion}

In the present study we identified the mitochondrial enzyme MSRB2 as a new interaction partner of the schizophrenia-associated protein LG72 using three different approaches. We also confirmed the co-localization of these two proteins in mitochondria of transiently transfected human and murine cell lines.

G72 is a gene that evolved late during the evolution of anthropoid primates. LG72 contains no recognizable protein motif and it is therefore impossible to deduce any functions from its sequence. Some findings indicated that LG72 binds to the D-serine degrading enzyme DAO (Chang et al. 2013; Chumakov et al. 2002; Sacchi et al. 2008; Sacchi et al. 2011). However, DAO and LG72 are localized in different subcellular compartments. DAO is clearly a peroxisomal protein, whereas there is no evidence for LG72 in peroxisomes. In contrast, several studies have shown a mitochondrial localization of LG72 (Kvajo et al. 2008; Otte et al. 2011; Sacchi et al. 2011). In order to reconcile the disparate sub-cellular localization with a putative modulation of DAO activity by LG72, it has been suggested that the two proteins interact in the cytosol during the transport of DAO into peroxisomes (Sacchi et al. 2011). Even if this were correct, we find it highly unlikely that modulation of DAO activity is the only function of LG72.

To identify other potential interaction partners of $L G 72$, we performed an $Y 2 H$ screen using LG72 as bait. We identified a total of 18 interacting proteins, among them the enzyme MSRB2. Since MSRB2 is located in mitochondria (Kim and Gladyshev 2004) - as is LG72 - we focused on this protein for further studies. The interaction between LG72 and MSRB2 was substantiated using the LUMIER-assay, coimmunoprecipitation experiments and co-localization studies.

MSRB2 is a member of the MSR protein family of detoxifying enzymes, which include the stereoselective methionine sulfoxide reductases MSRA and MSRB. These enzymes reduce methionine sulfoxides in proteins, which are generated by ROS (Moskovitz et al. 1996). MSRB2 is located in mitochondria in mammalian cells and catalyzes the reversion of the methionine R-sulfoxide to the reduced form of methionine (Kaya et al. 2010). It was demonstrated that silencing the expression of MSRB2 results in an exacerbated sensitivity toward oxidative stress (Ugarte et al. 
2013), while overexpression protects cells from oxidative stress (Cabreiro et al. 2008). Based on these findings and the results of our present study we suggest that LG72 expression affects the function of MSRB2.

Interestingly, knock-down of MSRB2 via siRNA resulted in specific expression changes of the glutathione S-transferase P1 (GSTP1), as demonstrated in a proteome analysis done in HEK-293 cells (Ugarte et al. 2013). GSTP1, an enzyme involved in the defense against oxidative stress, was also dysregulated in G72Tg mice, along with increased ROS production, mitochondrial dysfunctions, reduced GSH levels and increased malondialdehyde levels (Otte et al. 2011). According to this, both MSRB2 and LG72 seem to affect similar mechanisms involved in the cellular oxidative stress defense system. If LG72 is up-regulated, as has been observed in schizophrenia patients, MSRB2 function may be altered leading to increased mitochondrial oxidative stress and consequently to the damage of mitochondrial proteins. Such compromised mitochondrial function has indeed been found in schizophrenia patients (summarized in (Park and Park 2012)). In line with this, schizophrenic patients often display a number of biomarkers indicative of increased oxidative stress, including reduced glutathione (GSH) levels (Do et al. 2000), increased malondialdehyde levels (Zhang et al. 2006; Zhang et al. 2007), and altered antioxidant enzyme activities (Gysin et al. 2007; Zhang et al. 2006). Also, an impaired GSTP1 function has been associated with an increased risk to develop schizophrenia (Kashani et al. 2011). Together, mounting evidence implicates mitochondrial defects and the resulting oxidative stress in the pathogenesis of schizophrenia (Altar et al. 2005; Herken et al. 2001; Mahadik and Mukherjee 1996; Marchbanks et al. 2003; Prabakaran et al. 2004). Interestingly, polymorphisms in the mitochondrial MSRB3 have been associated with hippocampal volume and cognitive decline in a recent GWA study (Bis et al. 2012). MSRB3 is a paralog of MSRB2 that requires zinc as a cofactor.

In summary, we show for the first time that LG72 binds the mitochondrial enzyme MSRB2 and is co-localized with MSRB2 in mitochondria. This finding further supports a role of $L G 72$ in mitochondrial oxidative stress regulation. 


\section{Acknowledgements}

This work was supported by grants from the Federal Ministry of Education and Research (NGFN-MOODS FKZ 01GS08144).

\section{Conflict of interest}

All authors disclose no conflict of interest.

\section{References:}

Altar CA et al. (2005) Deficient hippocampal neuron expression of proteasome, ubiquitin, and mitochondrial genes in multiple schizophrenia cohorts Biol Psychiatry 58:85-96

Bis JC et al. (2012) Common variants at 12q14 and 12q24 are associated with hippocampal volume Nat Genet 44:545-551 doi:10.1038/ng.2237

Cabreiro F, Picot CR, Perichon M, Castel J, Friguet B, Petropoulos I (2008) Overexpression of mitochondrial methionine sulfoxide reductase B2 protects leukemia cells from oxidative stress-induced cell death and protein damage $\mathrm{J}$ Biol Chem 283:16673-16681 doi:10.1074/jbc.M708580200

Chang SL et al. (2013) The C-Terminal Region of G72 Increases d-Amino Acid Oxidase Activity International journal of molecular sciences 15:29-43 doi:10.3390/ijms15010029

Chumakov I et al. (2002) Genetic and physiological data implicating the new human gene G72 and the gene for D-amino acid oxidase in schizophrenia Proc Natl Acad Sci U S A 99:13675-13680 doi:10.1073/pnas.182412499

\section{9 [pii]}

Clay HB, Sillivan S, Konradi C (2011) Mitochondrial dysfunction and pathology in bipolar disorder and schizophrenia Int J Dev Neurosci 29:311-324 doi:S0736$\underline{5748(10) 00380-1 \text { [pii] }}$

\subsection{6/j.ijdevneu.2010.08.007}

Do KQ et al. (2000) Schizophrenia: glutathione deficit in cerebrospinal fluid and prefrontal cortex in vivo The European journal of neuroscience 12:3721-3728

Goldberg TE et al. (2006) The G72/G30 gene complex and cognitive abnormalities in schizophrenia Neuropsychopharmacology 31:2022-2032 doi:1301049 [pii] 10.1038/sj.npp.1301049 
Gysin R et al. (2007) Impaired glutathione synthesis in schizophrenia: Convergent genetic and functional evidence Proc Natl Acad Sci U S A 104:16621-16626

Herken H, Uz E, Ozyurt H, Sogut S, Virit O, Akyol O (2001) Evidence that the activities of erythrocyte free radical scavenging enzymes and the products of lipid peroxidation are increased in different forms of schizophrenia Mol Psychiatry 6:66-73 Kashani FL, Kordi-Tamandani DM, Sahranavard R, Hashemi M, Kordi-Tamandani F, Torkamanzehi A (2011) Analysis of glutathione S-transferase genes polymorphisms and the risk of schizophrenia in a sample of Iranian population Neuron glia biology 7:199-203 doi:10.1017/S1740925X12000130

Kaya A et al. (2010) Compartmentalization and regulation of mitochondrial function by methionine sulfoxide reductases in yeast Biochemistry 49:8618-8625 doi:10.1021/bi100908v

Kim HY, Gladyshev VN (2004) Methionine sulfoxide reduction in mammals: characterization of methionine-R-sulfoxide reductases Mol Biol Cell 15:1055-1064 doi:10.1091/mbc.E03-08-0629

Korostishevsky M et al. (2006) Transmission disequilibrium and haplotype analyses of the G72/G30 locus: suggestive linkage to schizophrenia in Palestinian Arabs living in the North of Israel Am J Med Genet B Neuropsychiatr Genet 141B:91-95 doi:10.1002/ajmg.b.30212

Kvajo M, Dhilla A, Swor DE, Karayiorgou M, Gogos JA (2008) Evidence implicating the candidate schizophrenia/bipolar disorder susceptibility gene $\mathrm{G} 72$ in mitochondrial function Mol Psychiatry 13:685-696 doi:4002052 [pii]

10.1038/sj.mp.4002052

Mahadik SP, Mukherjee S (1996) Free radical pathology and antioxidant defense in schizophrenia: a review Schizophr Res 19:1-17

Marchbanks RM, Ryan M, Day IN, Owen M, McGuffin P, Whatley SA (2003) A mitochondrial DNA sequence variant associated with schizophrenia and oxidative stress Schizophr Res 65:33-38

Moskovitz J, Jenkins NA, Gilbert DJ, Copeland NG, Jursky F, Weissbach H, Brot N (1996) Chromosomal localization of the mammalian peptide-methionine sulfoxide reductase gene and its differential expression in various tissues Proc Natl Acad Sci $U$ S A 93:3205-3208 
Mossner $\mathrm{R}$ et al. (2010) DAOA/G72 predicts the progression of prodromal syndromes to first episode psychosis Eur Arch Psychiatry Clin Neurosci 260:209-215 doi:10.1007/s00406-009-0044-y

Otte DM et al. (2011) N-acetyl Cysteine Treatment Rescues Cognitive Deficits Induced by Mitochondrial Dysfunction in G72/G30 Transgenic Mice Neuropsychopharmacology 36:2233-2243 doi:npp2011109 [pii]

10.1038/npp.2011.109

Palidwor GA et al. (2009) Detection of alpha-rod protein repeats using a neural network and application to huntingtin PLoS computational biology 5:e1000304 doi:10.1371/journal.pcbi.1000304

Park C, Park SK (2012) Molecular links between mitochondrial dysfunctions and schizophrenia Molecules and cells 33:105-110 doi:10.1007/s10059-012-2284-3

Petrakis S et al. (2012) Identification of human proteins that modify misfolding and proteotoxicity of pathogenic ataxin-1 PLoS genetics 8:e1002897 doi:10.1371/journal.pgen.1002897

Prabakaran S et al. (2004) Mitochondrial dysfunction in schizophrenia: evidence for compromised brain metabolism and oxidative stress Mol Psychiatry 9:684-697, 643 doi:10.1038/sj.mp.4001511

4001511 [pii]

Sacchi S et al. (2008) pLG72 modulates intracellular D-serine levels through its interaction with D-amino acid oxidase: effect on schizophrenia susceptibility $\mathrm{J}$ Biol Chem 283:22244-22256 doi:M709153200 [pii]

10.1074/jbc.M709153200

Sacchi S, Cappelletti P, Giovannardi S, Pollegioni L (2011) Evidence for the interaction of D-amino acid oxidase with pLG72 in a glial cell line Mol Cell Neurosci 48:20-28 doi:10.1016/j.mcn.2011.06.001

Schumacher $\mathrm{J}$ et al. (2004) Examination of G72 and D-amino-acid oxidase as genetic risk factors for schizophrenia and bipolar affective disorder Mol Psychiatry 9:203-207 doi:10.1038/sj.mp.4001421

4001421 [pii]

Stelzl U et al. (2005) A human protein-protein interaction network: a resource for annotating the proteome Cell 122:957-968 doi:10.1016/j.cell.2005.08.029

Ugarte N, Ladouce R, Radjei S, Gareil M, Friguet B, Petropoulos I (2013) Proteome alteration in oxidative stress-sensitive methionine sulfoxide reductase-silenced 
HEK293 cells Free Radic Biol Med 65:1023-1036 doi:10.1016/j.freeradbiomed.2013.08.008

Zhang XY, Tan YL, Cao LY, Wu GY, Xu Q, Shen Y, Zhou DF (2006) Antioxidant enzymes and lipid peroxidation in different forms of schizophrenia treated with typical and atypical antipsychotics Schizophr Res 81:291-300

Zhang XY et al. (2007) Disrupted antioxidant enzyme activity and elevated lipid peroxidation products in schizophrenic patients with tardive dyskinesia The Journal of clinical psychiatry 68:754-760

Zhang Z et al. (2009) First evidence of association between $G 72$ and bipolar disorder in the Chinese Han population Psychiatr Genet 19:151-153 doi:10.1097/YPG.0b013e32832a50f1

Zuliani R et al. (2009) Genetic variation in the G72 (DAOA) gene affects temporal lobe and amygdala structure in subjects affected by bipolar disorder Bipolar Disord 11:621-627 doi:BDI731 [pii] 10.1111/j.1399-5618.2009.00731.x 
A)

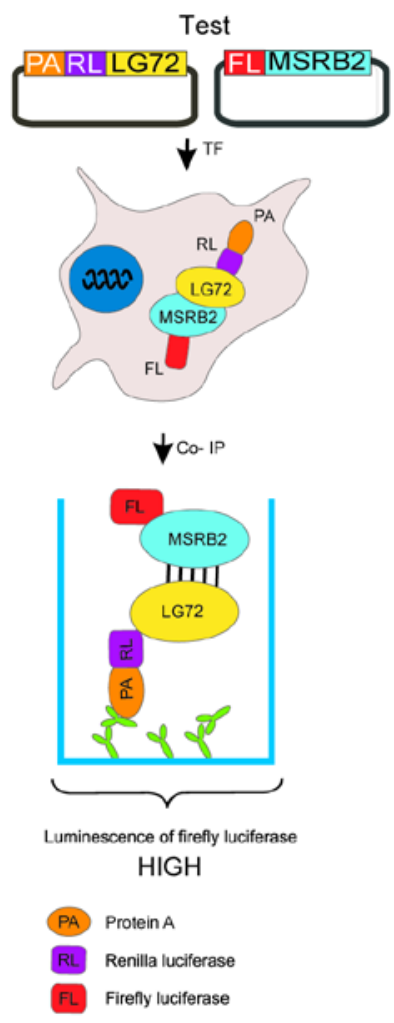

B)

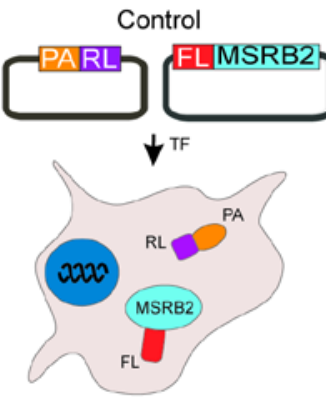

$\downarrow$ Co.IP

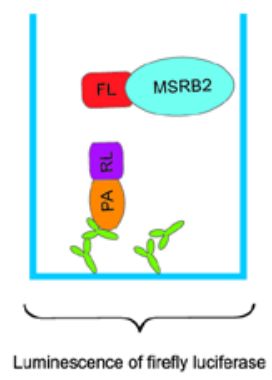

LOW

$\mathrm{R}-\mathrm{op}=\frac{\mathrm{FLS} \text { (Test) }}{\mathrm{FLS} \text { (Control) }} \geq 1.2$

C)
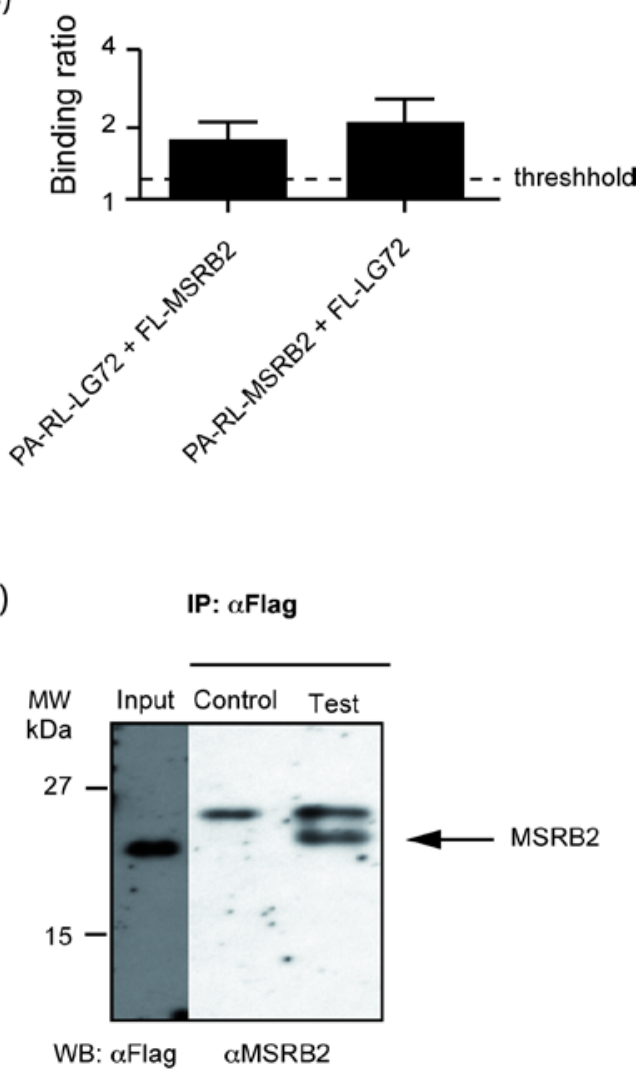

Fig. 1) LG72 interacts with MSRB2 (a) Schematic representation of the LUMIER co-immunoprecipiation assay. The protein A (PA)-Renilla luciferase ( $R L)$-tagged bait (PA-RL-LG72) and the firefly luciferase (FL)-tagged prey protein (FL-MSRB2, modifier) were co-expressed in HEK-293 cells. After co-immunoprecipitation from cell lysates the interaction between bait and prey fusion proteins was monitored by quantification of firefly luciferase activity in protein complexes.

(b) The human proteins LG72 and MSRB2 specifically interact with each other in LUMIER co-immunoprecipitation assays. An R-op binding ratio of $\geq 1.2$ (dotted line) indicates the specificity of interaction in mammalian cells.

(c) HEK-293 cells were transfected with a LG72-Flag-expressing construct. After 72 hours, the cells were subjected to anti-FLAG co-immunoprecipitation assays. Immunoprecipitated LG72-Flag and co-immunoprecipitated MSRB2 was determined by anti-Flag and anti-MSRB2 immunoblots, respectively. The input sample indicates the amount of LG72-Flag protein used for the immunoprecipitation. The asterisk indicates a non-specific band that was also present in the control sample.

Abbreviations: PA - protein A tag; RL - Renilla luciferase; $F L$ - firefly luciferase; MSRB2 - methionine S reductase B2 - prey protein; TF - transfection of cells; IP _ immunoprecipitation; FLS (A) - firefly luciferase signal. 

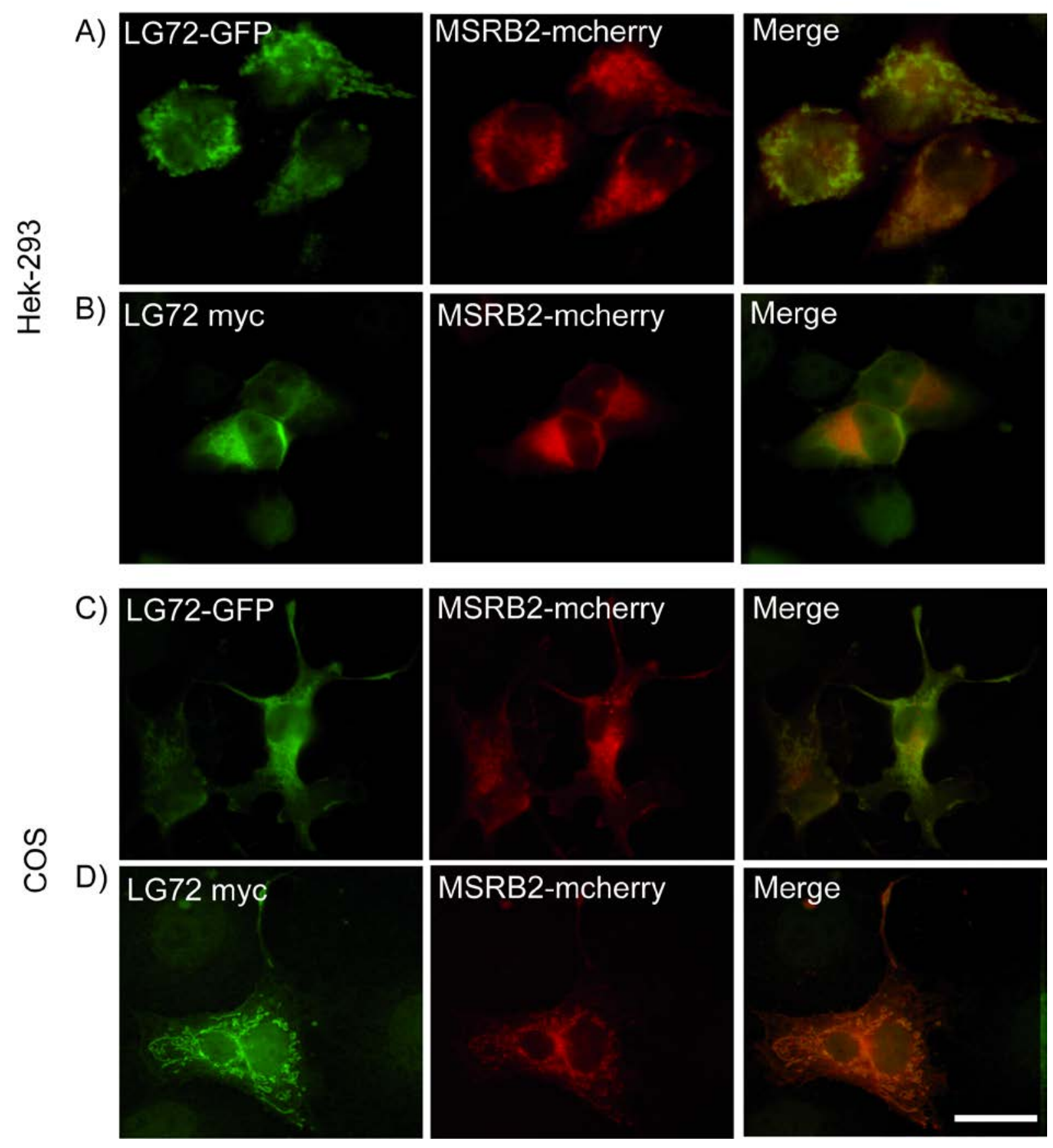

Fig. 2) LG72 co-localizes with MSRB2 Fluorescence microscopic analysis of the subcellular localization of GFP or myc-tagged LG72 (green) or mCherry-tagged MSRB2 (red). (a, c): HEK-293 or COS cells co-transiently transfected with CMVG72-GFP and CMV-MSRB2-mCherry. (b, d): HEK-293 / COS cells transiently cotransfected with CMV-G72-myc and CMV-MSRB2-mCherry, then stained with antimyc antibody and Alexa Fluor 488 linked secondary antibody. Yellow areas in the merged pictures represent regions of co-localization. Scale bar: $25 \mu \mathrm{m}$. 
$\mathrm{N} 2 \mathrm{a}$
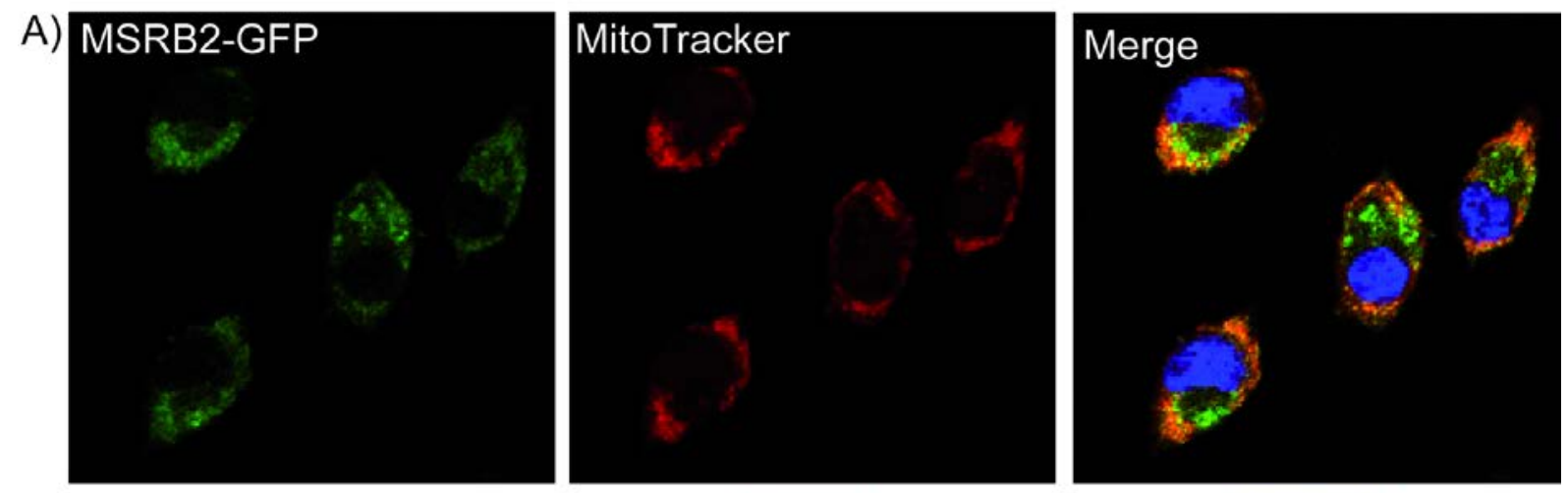

B) LG72-GFP
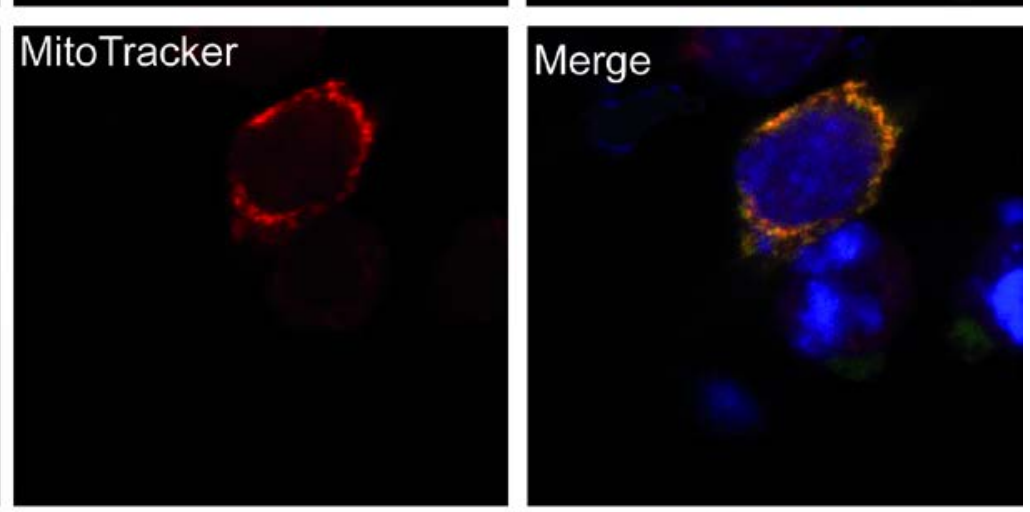

C) LG72-GFP
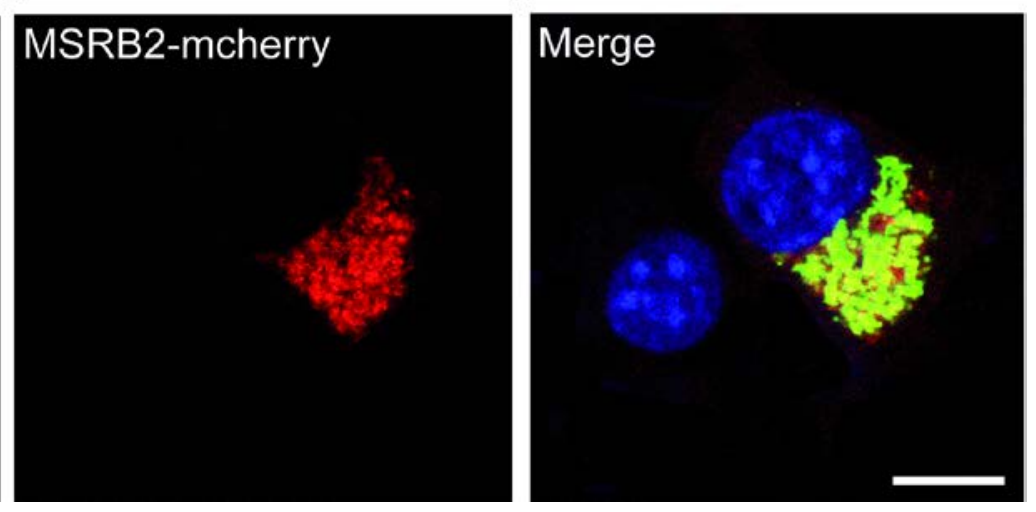

Fig. 3) LG72 is a mitochondrial protein and co-localizes with MSRB2 in N2a cells (a): N2a cells transiently transfected with a CMV-MSRB2-GFP construct and stained with MitoTracker®. (b): N2a cells transiently transfected with a CMV-G72GFP construct and stained with MitoTracker®. (c): N2a cells transiently cotransfected with CMV-LG72-GFP and CMV-MSRB2-mCherry constructs. Yellow areas in the merged pictures represent regions of co-localization. Scale bar: $15 \mu \mathrm{m}$. 